\title{
The determination of glacier speed by time-lapse photography under unfavorable conditions
}

\author{
W. D. Harrison, K. A. Echelmeyer, D. M. Cosgrove, \\ Geophysical Institute, University of Alaska, Fairbanks, Alaska 99775-0800, U.S.A. \\ C. F. RAYMOND \\ Geophysics Program AK-50, University of Washington, Seattle, Washington 98195, U.S.A.
}

\begin{abstract}
Two practical problems in the use of time-lapse photography for the measurement of speed were encountered during the recent surge of West Fork Glacier in the central Alaska Range, Alaska, U.S.A. The first is severe rotational camera instability; we show how natural, unsurveyed features on the valley wall can be used to make the necessary corrections. The second problem is the computation of absolute speed when many different, unsurveyed glacier-surface features are used as targets. We give a method for connecting the data obtained from different targets, and for determining the scale using limited information obtained by surveying. Severe systematic errors can occur unless the angle between the axis of the lens and the direction of horizontal motion is determined.
\end{abstract}

\section{INTRODUGTION}

Time-lapse photography is a powerful technique for obtaining detailed information about glacier speed and its variations, but in our experience it must often be carried out under less than ideal conditions. For example, during the recent surge of West Fork Glacier in the central Alaska Range, Alaska, U.S.A. (Echelmeyer and Harrison, 1989), we were forced to deploy cameras at sites chosen for their accessibility by light aircraft and not for their suitability as stable platforms for cameras. At one of these sites, designated as "upper camera", access was particularly marginal. During our several visits to the site, aircraft problems usually absorbed more attention than the camera (which was installed on an unstable platform of snow, ice and rock), than the targets (small air-dropped spruce trees which were neither surveyed nor found in the photographs) and than the installation and surveying of fixed control points (which were never seriously considered). The almost inevitable result of such a marginal operation is marginal data, and the problem of reducing them to absolute glacier velocity, which is not a trivial problem under the best of conditions, is a major challenge.

Here we describe some aspects of the reduction of the data from the "upper camera" and "lower camera" sites on West Fork Glacier. There are two problems: first, that of removing the effects of camera instability (by this we mean instability in the orientation of the camera) and, secondly, that of determining the glacier speed after the effects of instability are removed. Both of these problems had to be solved using natural unsurveyed features, both as reference points on the valley walls and as moving targets on the glacier surface. In previous applications we have not made corrections for camera instability, and have usually had the benefit of sharply defined and periodically surveyed moving targets. However, three other critical pieces of information were available for reduction of the West Fork Glacier data: the camera orientation at the time of installation, the direction of glacier motion and the glacier speed at one time.

A photograph, or "frame", typical of those obtained in good weather, is shown in Figure 1. One frame was taken per day by each camera. Each frame was projected from its negative on to a digitizing table in enlarged form, and the positions of the edges of the frame and of the images of the chosen features were measured. Care was taken not to move the projector or digitizing table between measurements on different frames. These data were then scaled to the standard frame size, which was known from direct measurements of a few frames made with a measuring microscope. More details have been given by Harrison and others (1986). The coordinates of the images were expressed in a system centered in the plane of the film with the axes parallel to the edges of the frame. These "film-plane coordinates", or more precisely, these coordinates divided by the focal length, are the basic input data to our analysis.

It is worth noting that the problem of measuring glacier speed by time-lapse photography is rather different from that of map making by photogrammetry. In the latter case, high absolute accuracy is required, and consequently high quality, suitably calibrated metric cameras are used. In the former case, the change in position of an image between successive frames is of primary concern, and minor distortions due to imperfections in the interior elements of the camera (or in the 


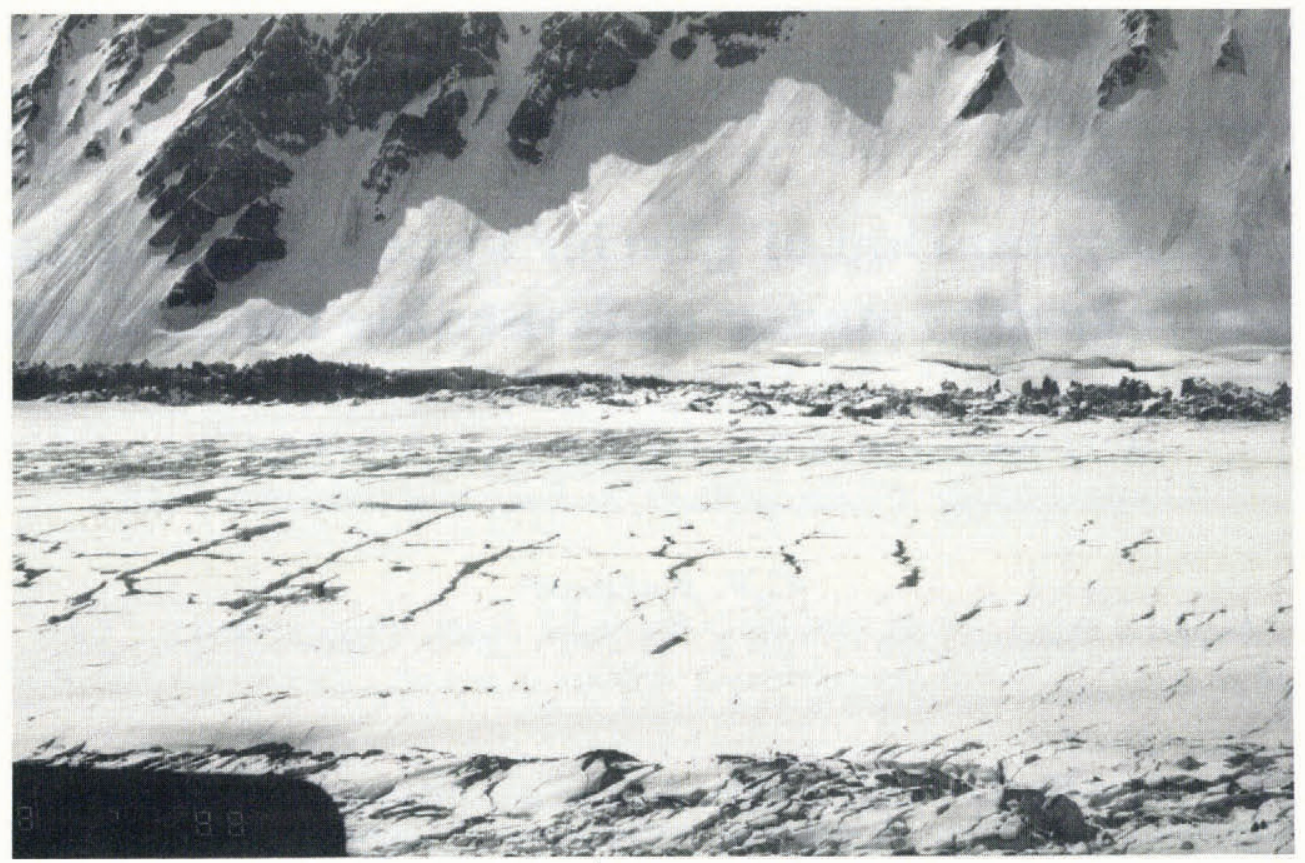

Fig. 1. Frame taken at lower camera, day 129 1988. The glacier flows from left to right.

projection and measuring systems used to analyze the photographs) are not of great importance, as long as they do not change abruptly from one frame to the next. Because of these limited requirements, inexpensive off-theshelf $35 \mathrm{~mm}$ cameras turn out to be adequate for our applications. The main problem with our cameras (and probably with any camera in unattended operation) is not optics but reliability. Automatic cameras are inherently complex electro-mechanical devices and, as soon as they are left to themselves, they tend not to work. Over the years we have observed at least a dozen different failure modes, a few of which we have been able to cure by modifying the cameras and our operating procedures.

Other applications of time-lapse photography to the measurement of glacier motion have been discussed by Flotron (1973), Krimmel and Rasmussen (1986) and Harrison and others (1986). The methods of Krimmel and Rasmussen are of most interest to this paper, because the basic problems which they faced were similar to ours: camera rotational instability and the intersection of rays with surfaces to determine target motion. One important difference is that the coordinates of the fixed reference points used to correct for changes in camera orientation were known to them but not to us. Another difference is that they also used fixed points as control to make corrections for small differences in magnification between successive frames, including the effects of differing enlargement factors in the photographic prints upon which they made their measurements. Our data-reduction procedures described above made the analogous corrections without the use of fixed points, and no significant improvement could be obtained with the quality of fixed-point control available to us.

\section{BASIC THEORY}

The purpose of a camera is the same as that of a theodolite: to measure the horizontal and vertical angles which define the path of a ray of light from a target to the instrument. A camera accomplishes this by recording the coordinates $(\xi, \zeta)$ of the target image as measured in the plane of the film, the film-plane coordinates. To obtain the horizontal and vertical angles with a theodolite, one must not only point and read the instrument but also determine its orientation. This is done by leveling it in the two horizontal directions and by measuring the horizontal angle with respect to a reference point, a procedure which is equivalent to determining three angles which define the orientation of the theodolite. Similarly, to obtain the horizontal and vertical angles with a camera, one must determine three angles $(\theta, \phi, \rho)$ which define the orientation of the camera. Unfortunately, these orientation angles change between the exposure of successive frames if the camera is unstable, and they must be calculated for each frame using the measured film-plane coordinates $(\xi, \zeta)$ of fixed reference points. Once this problem has been solved, a set of film-plane coordinates that is corrected for camera instability can be calculated, and the problem of relating them to the glacier speed can be addressed.

The solution of these problems requires a pair of basic relationships which expresses the two film-plane coordinates of the target image in terms of the camera coordinates, the coordinates $(x, y, z)$ of the actual target and the angles $(\theta, \phi, \rho)$ which define the orientation of the camera. This is a standard problem in photogrammetry (see Wolfe, 1983; Krimmel and Rasmussen, 1986; for example) but, for the sake of completeness, we give a brief derivation here in a form which is convenient for our applications.

These required relationships are determined by the camera optics, in a way which is simple when expressed in a coordinate system with its origin at the camera lens, and oriented with one axis coinciding with the axis of the camera lens and the other two axes parallel to the edges of the frame. (We denote this coordinate system by triple primes). The first step in the derivation is therefore to find 


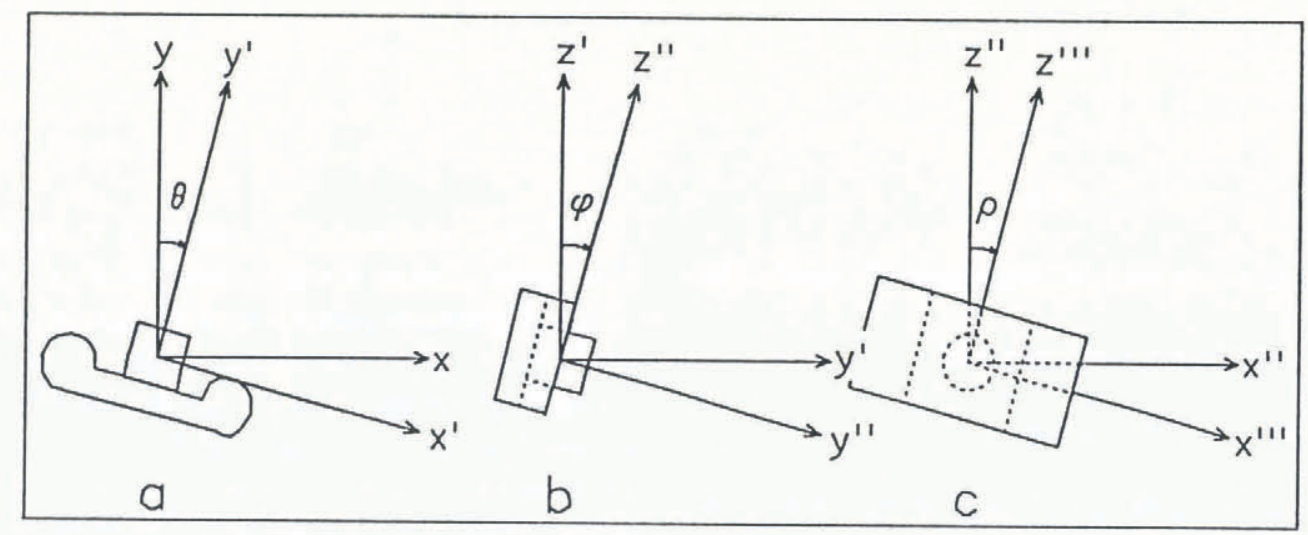

Fig. 2. The act of rotating the camera into a general orientation specified by the three angles $(\theta, \phi, \rho)$, as decomposed into three separate rotations: (a) Set azimuth, rotate about z-axis. This defines a new coordinate system denoted by single primes. (b) Set inclination, rotate about $x^{\prime}$-axis. This defines the double-prime coordinate system. (c) Set axial orientation, rotate about $y^{\prime \prime}$-axis. This defines the triple-prime system.

the coordinates $\left(x^{\prime \prime \prime}, y^{\prime \prime \prime}, z^{\prime \prime \prime}\right)$ of a target in such a system, given their values $(x, y, z)$ in a reference system, where, for example, the $x$ - and $y$-axes might be chosen to lie in the horizontal plane. The coordinate systems are orthogonal and righthanded, and their origins coincide with the camera lens. The transformation is obtained by imagining the camera to be pointed initially along the $y$-axis, with the $x$ - and $z$-axes parallel to the edges of the frame, and then to be rotated into some general orientation by three successive rotations referenced to a sequence of coordinate systems as shown in Figure 2a, b and c. The third step allows for rotation of the camera about the axis of the lens. The result is

$$
\begin{aligned}
\left(\begin{array}{l}
x^{\prime \prime \prime} \\
y^{\prime \prime \prime} \\
z^{\prime \prime \prime}
\end{array}\right)= & \left(\begin{array}{ccc}
\cos \rho & 0 & -\sin \rho \\
0 & 1 & 0 \\
\sin \rho & 0 & \cos \rho
\end{array}\right)\left(\begin{array}{ccc}
1 & 0 & 0 \\
0 & \cos \phi & -\sin \phi \\
0 & \sin \phi & \cos \phi
\end{array}\right) \\
& \left(\begin{array}{ccc}
\cos \theta & -\sin \theta & 0 \\
\sin \theta & \cos \theta & 0 \\
0 & 0 & 1
\end{array}\right)\left(\begin{array}{l}
x \\
y \\
z
\end{array}\right)
\end{aligned}
$$

Note that we have made the $y^{\prime \prime \prime}$-axis coincide with the axis of the lens. It is recalled that the angles $(\theta, \phi, \rho)$ describe the orientation of the camera with respect to the axes of the $(x, y, z)$ coordinate system as shown in Figure 2.

The effect of camera optics is that a point target, the point $\left(x^{\prime \prime \prime}, y^{\prime \prime \prime}, z^{\prime \prime \prime}\right)=(0,0,0)$ (which is at the center of the lens) and the image of the target are collinear. (A more rigorous statement which allows for the finite thickness of the lens leads to the same results.) The image of the target lies in the plane of the film, which is a distance $f$ behind the lens, where $f$ is the focal length. Collinearity is then expressed by

$$
\begin{aligned}
& \frac{x^{\prime \prime \prime}}{y^{\prime \prime \prime}}=-\frac{x^{\prime \prime \prime}(\mathrm{neg})}{f}=\frac{x^{\prime \prime \prime}(\mathrm{pos})}{f} \equiv \xi \\
& \frac{z^{\prime \prime}}{y^{\prime \prime \prime}}=-\frac{z^{\prime \prime}(\mathrm{neg})}{f}=\frac{z^{\prime \prime \prime}(\mathrm{pos})}{f} \equiv \zeta
\end{aligned}
$$

where $\left(x^{\prime \prime \prime}, y^{\prime \prime \prime}, z^{\prime \prime \prime}\right)$ are the coordinates of the target point, and $x^{\prime \prime \prime}\left(\right.$ neg), $z^{\prime \prime \prime}($ neg) are the coordinates of the image point (which is in the plane of the film), measured from the center of the frame (where the axis of the lens intersects the film plane) along coordinate axes parallel to the edges of the frame. $x^{\prime \prime \prime}$ (pos), $z^{\prime \prime \prime}$ (pos) are the same quantities, but measured on an erect and unreversed view of the scene. $(\xi, \zeta)$ are these quantities scaled by the focal length, and they are the only film-plane quantities needed in the theory, since neither the unscaled film-plane coordinates nor the focal length occurs alone. We therefore define $(\xi, \zeta)$ to be "the film-plane coordinates" in what follows. It is convenient to call these the horizontal and vertical filmplane coordinates, respectively, since the edges of the frames have approximately these orientations in our applications.

Combination of Equation (1) with Equation (2) gives

$$
\begin{gathered}
\xi=[(X \cos \theta-\sin \theta) \cos \rho \\
-\{(X \sin \theta+\cos \theta) \sin \phi+Z \cos \phi\} \sin \rho] \\
/[(X \sin \theta+\cos \theta) \cos \phi-Z \sin \phi] \\
\zeta=[(X \cos \theta-\sin \theta) \sin \rho \\
+\{(X \sin \theta+\cos \theta) \sin \phi+Z \cos \phi\} \cos \rho] \\
/[(X \sin \theta+\cos \theta) \cos \phi-Z \sin \phi]
\end{gathered}
$$

where

$$
(X, Z) \equiv\left(\frac{x}{y}, \frac{z}{y}\right) .
$$

It is recalled that $(x, y, z)$ are the coordinates of the target in a system in which the camera lens is at the origin. The ratios $(x / y, z / y)$ define the direction of a ray between the target and the camera, and are therefore analogous to the horizontal and vertical angles measured by a theodolite if the $x$ - and $y$-axes are chosen to lie in the horizontal plane. These angles are $(\arctan X, \arctan Z)$, respectively.

The inverse of Equations (3), which can be found directly but is more easily obtained by inverting Equation (1) and combining the results with Equations (2), is 


$$
\begin{aligned}
& X=[(\xi \cos \rho+\zeta \sin \rho) \cos \theta \\
&+\{\cos \phi+(-\xi \sin \rho+\zeta \cos \rho) \sin \phi\} \sin \theta] \\
& / {[-(\xi \cos \rho+\zeta \sin \rho) \sin \theta} \\
&+\{\cos \phi+(-\xi \sin \rho+\zeta \cos \rho) \sin \phi\} \cos \theta], \\
& Z=[-\sin \phi+(-\xi \sin \rho+\zeta \cos \rho) \cos \phi] \\
& / {[-(\xi \cos \rho+\zeta \sin \rho) \sin \theta} \\
&+\{\cos \phi+(-\xi \sin \rho+\zeta \cos \rho) \sin \phi\} \cos \theta] .
\end{aligned}
$$

In Figure 2 the sequence of operations used to define the orientation of the axis of the lens and the edges of the frame with respect to the axes of the $(x, y, z)$ coordinate system is rather arbitrary. In the special case in which the $x$ - and $y$-axes are in the horizontal plane, our first two angles, $\theta$ and $\phi$, correspond to the horizontal and vertical scales which define the orientation of the telescopes on most theodolites (except that $\phi$ is measured from the horizontal rather than the vertical). Thus, our conventions make the pointing of a camera similar to the pointing of a theodolite. They are not the conventions commonly used in photogrammetry or by Krimmel and Rasmussen (1986).

\section{THE PROBLEM OF GAMERA INSTABILITY}

As already discussed, the purpose of a camera is to determine the quantities $(X, Z)$, defined by Equation (4) and analogous to the two angles measured by a theodolite, in terms of the measured film-plane coordinates $(\xi, \zeta)$ and the camera orientation angles $(\theta, \phi, \rho)$. Equations (5) permit this to be done for a target in any frame of interest if one measures $(\xi, \zeta)$ and can determine the angles $(\theta, \phi, \rho)$ existing at the time of exposure. The latter must be calculated separately for each frame when the camera is unstable. We shall show how this can be done using the measured $(\xi, \zeta)$ of fixed reference points; the desired $(X, Z)$ of the target of primary interest can then be calculated from Equations (5). (It is worth noting that camera translation between the times of exposure of successive frames, as opposed to rotation, can be ignored, because it is typically three orders of magnitude less than the motion of the glacier.)

A useful simplification (which is used temporarily and only for the purpose of making the corrections for camera instability) is to choose the orientation of the $(x, y, z)$ coordinate system such that $(\theta, \phi, \rho)=(0,0,0)$ at the time of exposure of the first frame; then the $(X, Z)$ in all subsequent frames refers to this coordinate system. From Equations (5), it is seen that in this coordinate system

* The correspondence between our angles and film-plane coordinates, and those of Krimmel and Rasmussen (denoted by the subscript KR), is as follows:

$\theta=\frac{\pi}{2}-\theta_{\mathrm{KR}}, \phi=-\phi_{\mathrm{KR}}, \rho=-\psi_{\mathrm{KR}}, \xi=\frac{\lambda_{\mathrm{i}_{\mathrm{KR}}}}{\sigma_{\mathrm{KR}}}$ and $\zeta=\frac{\mu_{\mathrm{i}_{\mathrm{KR}}}}{\sigma_{\mathrm{KR}}}$.
$(X, Z)=(\xi, \zeta)$. Therefore, the $(X, Z)$ for subsequent frames obtained by this choice of coordinate system are a set of film-plane coordiantes corrected to the camera orientation existing at the time of exposure of the first frame.

Using this coordinate system, it is possible to calculate the angles $(\theta, \phi, \rho)$ for each frame from the measured $(\xi, \zeta)$ of fixed reference points. A point that is fixed has constant $(X, Z)$ in all frames and, because of our choice of coordinate system, this $(X, Z)$ equals the $(\xi, \zeta)$ measured in the first frame. At least two fixed points are required because there are three unknown angles, and because each point gives only two pieces of information (its film-plane coordinates). We use two fixed points (because it is often difficult to find more) and calculate $(\theta, \phi, \rho)$ for each frame by a method of non-linear least squares based on Equations (3), using a program employing the Levenberg-Marquardt algorithm (Press and others, 1986). With $(\theta, \phi, \rho)$ thus determined, Equations (5) are used to calculate the corrected film-plane coordinates of the moving-glacier target, which, as we have stated, are simply $(X, Z)$ because of the choice of coordinate system.

The effectiveness of this procedure for finding a set of corrected film-plane coordinates is illustrated with a sample of data from the wildly unstable upper camera, which had a $35 \mathrm{~mm}$ format (frame size $24 \mathrm{~mm}$ by $36 \mathrm{~mm}$ ) and a focal length of $100 \mathrm{~mm}$. The camera-orientation angles are shown as functions of time in Figure 3. The camera is initially stable, becomes extremely unstable (probably as the spring thaw commences) and stabilizes again a month later (probably when the thaw is complete). Although not shown in Figure 3, it becomes unstable again about 1 September (probably with the onset of freezing). The instability with respect to rotation $\rho$ about the axis of the lens is particularly large. Instability was probably increased by the practical necessity of mounting the camera on a tripod on the surface rather than on some kind of monopod set into the ground, which tends to be more satisfactory. A sample of corrected and

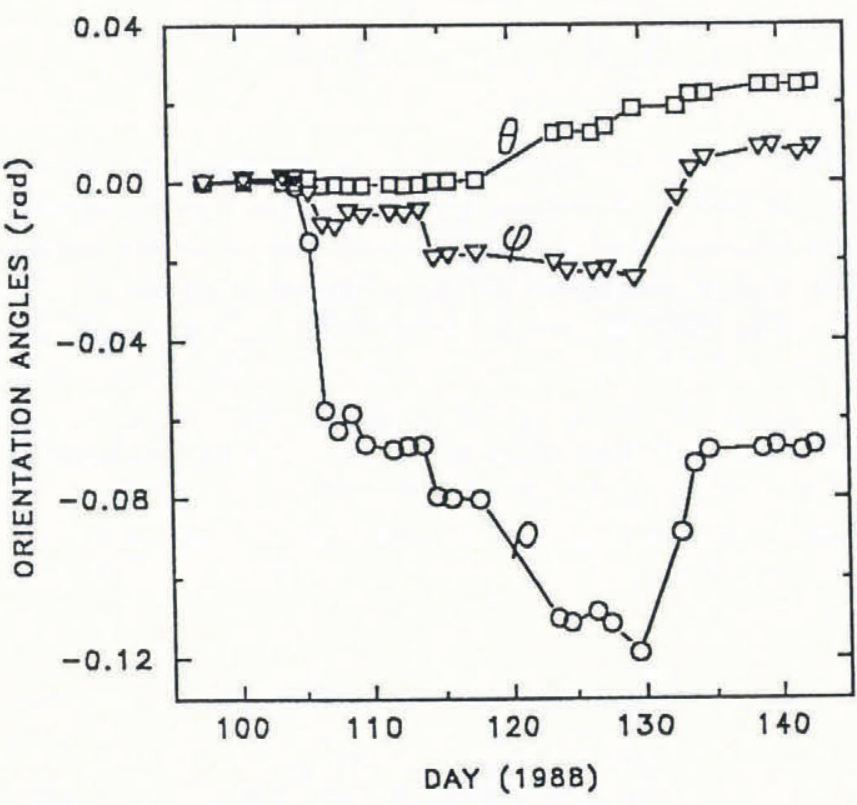

Fig. 3. Calculated orientation angles for upper camera as functions of time. The angles are defined in Figure 1. 


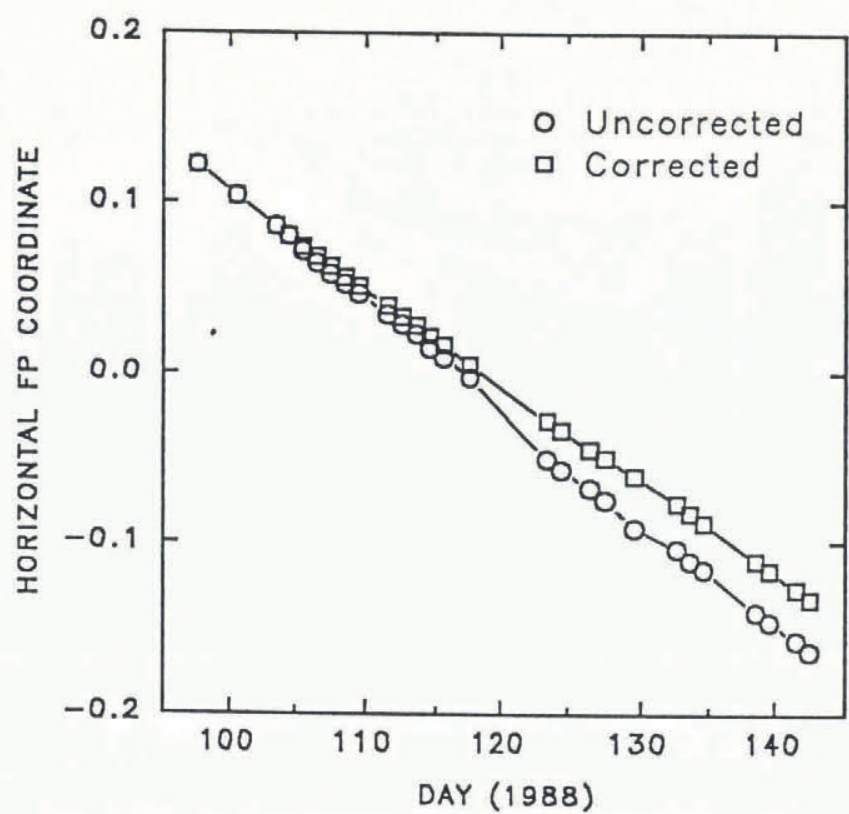

Fig. 4. Horizontal film-plane coordinate $\xi$ of a moving glacier target at upper camera, uncorrected and corrected for camera instability. The film-plane coordinates are dimensionless, since they are scaled by the focal length (Equations (2)).

uncorrected horizontal film-plane coordinates of a moving-glacier target is shown in Figure 4, and the corresponding film-plane speeds, defined as $\mathrm{d} \xi / \mathrm{d} t$ and evaluated by numerical differentiation, are shown in Figure 5. The corrected and uncorrected vertical filmplane coordinates are shown in Figure 6.

The random error is controled by the consistency with which the natural features used both for the fixed reference points and for the moving-glacier targets can be identified in successive frames. We estimate that the resulting error in the corrected horizontal film-plane coordinate $\xi$ is somewhat less than $4 \times 10^{-4}$. This corresponds to roughly $\frac{1}{2} \mathrm{~m}$ in target position when it is calculated from the corrected film-plane coordinates (it depends mainly upon $\xi)$, as described in the next section. This estimate is made from the scatter in the resulting speed of the glacier, and it is not considered accurate because it is difficult to separate observational effects from real variations in speed. The uncertainty is not small, but it is acceptable because motion of the glacier is large, roughly $7 \mathrm{~m}$ at upper camera, over the $1 \mathrm{~d}$ interval between successive frames. From previous experience, we expect, given the distance of roughly $1300 \mathrm{~m}$ from the camera to the targets, that the accuracy would have been about an order of magnitude greater if it had been feasible to use well-defined, Manmade markers for the reference points and the moving targets. On the other hand, the determination of the corrected set of film-plane coordinates does not require that the positions of the fixed reference points be known, and nothing would be gained by surveying them.

\section{THE PROBLEM OF SPEED}

We next consider the problem of calculating the velocity components of the glacier from the film-plane coordinates,
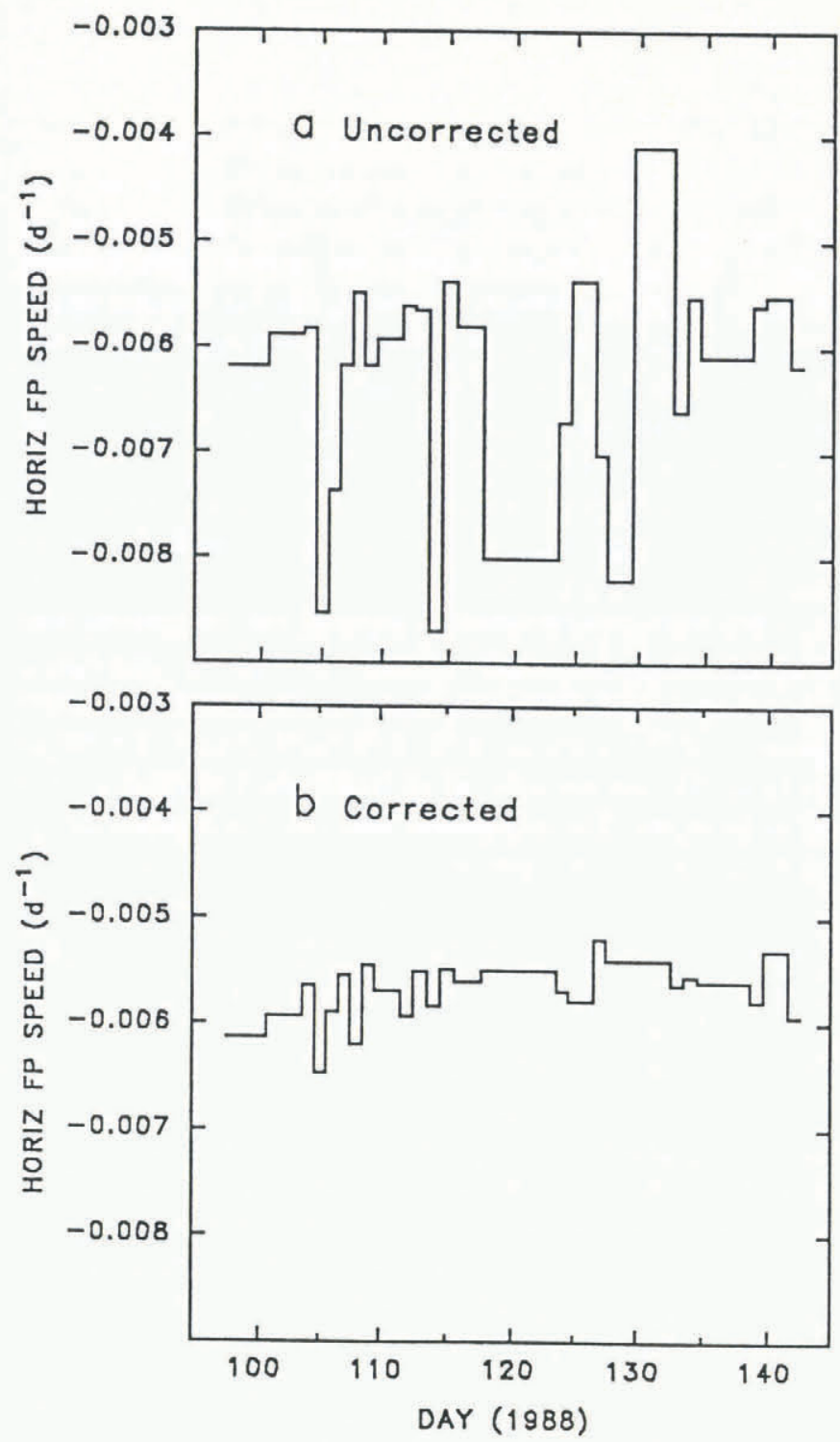

Fig. 5. Horizontal film-plane speed $\mathrm{d} \xi / \mathrm{d} t$ of a moving glacier target at upper camera: (a) Uncorrected. (b) Corrected for camera instability.

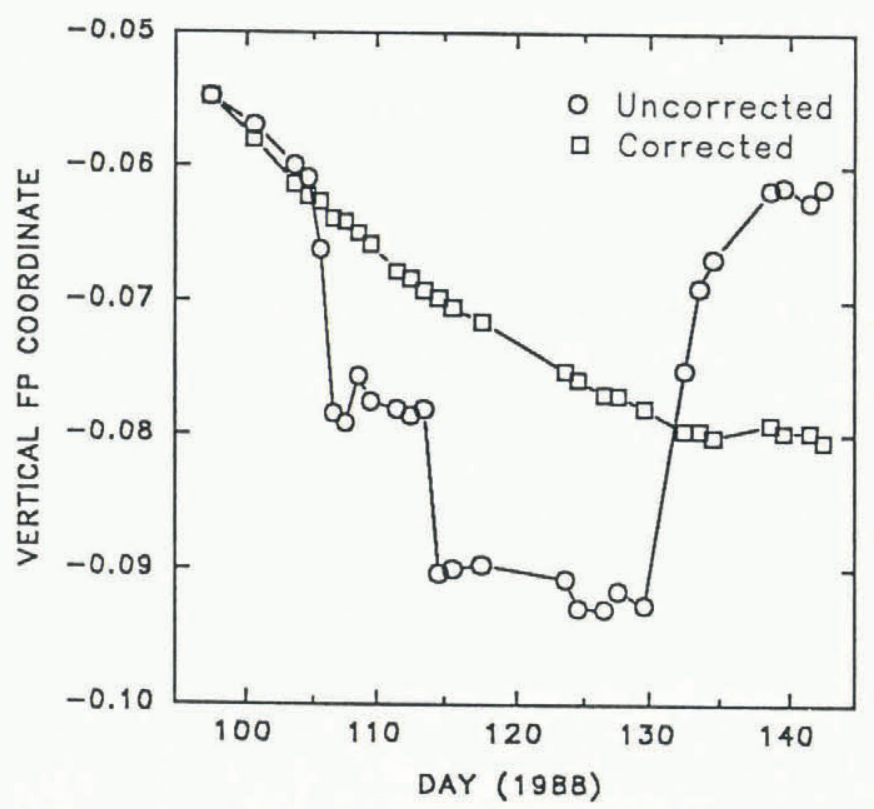

Fig. 6. Vertical film-plane coordinate $\zeta$ of a moving glacier target at upper camera, uncorrected and corrected for camera instability. 
assuming that the latter have been corrected for camera instability as just described. This is not an easy task in the case of West Fork Glacier. Many different natural surface features were used as targets (usually with some overlap), new ones being chosen as old ones were lost or passed out of the field of view. These targets were not located at exactly the same distance from the camera, and none of them was ever surveyed. However, the horizontal speed was measured directly by optical surveying once in the vicinity of the lower camera targets, and some information about camera orientation and direction of glacier motion was available. To interpret these data in terms of velocity, we need to express velocity in terms of the film-plane coordinates, the trajectory of the target and the orientation angles of the camera.

Because we now wish to separate the horizontal and vertical components of velocity, we use a coordiante system in which the $x$ - and $y$-axes lie in the horizontal plane, and the $z$-axis is vertical. We still require the origin to be at the camera lens, in order for the theory of section II to be valid. The horizontal and vertical components of velocity $(u, w)$ are given by

$$
\begin{aligned}
& u=\left[\left(\frac{\mathrm{d} x}{\mathrm{~d} t}\right)^{2}+\left(\frac{\mathrm{d} y}{\mathrm{~d} t}\right)^{2}\right]^{\frac{1}{2}} \\
& w=\frac{\mathrm{d} z}{\mathrm{~d} t} .
\end{aligned}
$$

$(u, w)$ are uniquely determined by a single camera only if we specify the surfaces in which the targets move. The simplest assumption, that these are vertical planes, seems valid because the glacier is reasonably straight on the scale of the field of view (about $500 \mathrm{~m}$ ), especially at lower camera. These planes are represented by

$$
y=m x+b .
$$

$m$ specifies the direction of motion and $b$ is related to the distance of the plane of motion from the camera, as discussed later. Both $m$ and $b$ are assumed to be independent of time. $m$ is also assumed to be the same for all targets (which is reasonable because most lie within a few hundred meters of each other), but $b$ is different for each target. Equation (7) permits Equation (6a) to be rewritten as

$$
u=\left(1+m^{2}\right)^{\frac{1}{2}} \frac{\mathrm{d} x}{\mathrm{~d} t} .
$$

Equations (6b) and (8) reduce the problem to that of expressing two of the target coordinates $(x, z)$ in terms of the film-plane coordinates. To do this, we use Equations (4) and (7) to write

$$
\begin{aligned}
& x=b \frac{X}{1-m X} \\
& z=b \frac{Z}{1-m X} .
\end{aligned}
$$

Equations (8), (6b) and (9) give

$$
\begin{aligned}
& u=b\left(1+m^{2}\right)^{\frac{1}{2}} \frac{\mathrm{d}}{\mathrm{d} t}\left(\frac{X}{1-m X}\right) \\
& w=b \frac{\mathrm{d}}{\mathrm{d} t}\left(\frac{Z}{1-m X}\right)
\end{aligned}
$$

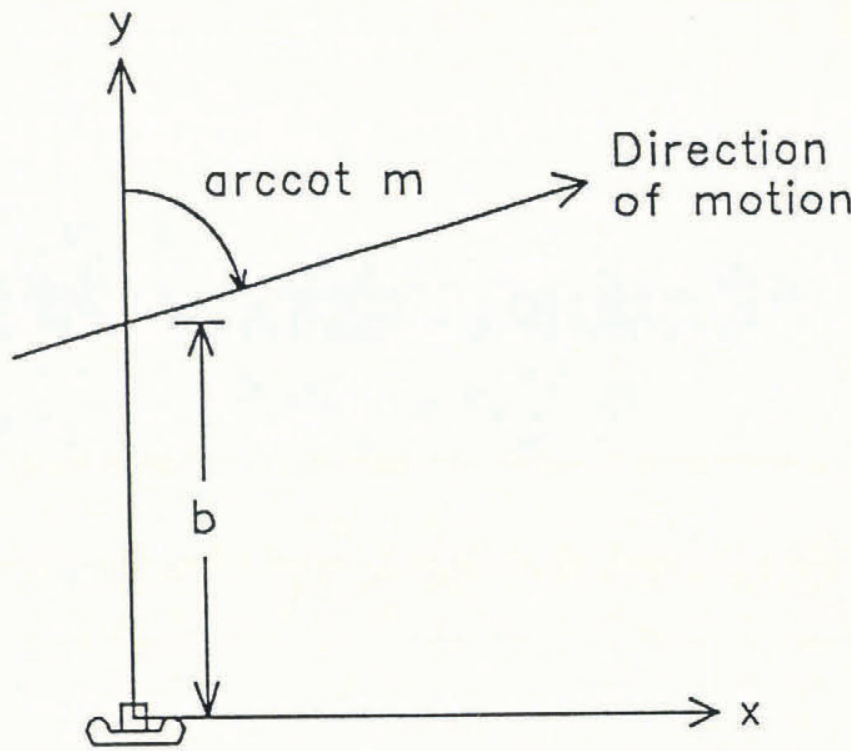

Fig. 7. Relationship between the orientation of the camera and the direction of glacier motion, when the orientation of the $y$ axis is chosen such that $\theta=0$. The $x$-and $y$-axes are in the horizontal plane. The axis of the lens and the $y$-axis lie in the plane $x=0$; these axes coincide only if the inclination angle $\phi=0$. The motion vector lies in the plane $y=m x+b$. The intersection angle between the planes is arccot $m$.

which are the desired relationships between the velocity components of a moving-glacier target and the film-plane coordinates, since $(X, Z)$ in Equations (10) can be expressed in terms of the film-plane coordinates by Equations (5). As long as we keep the $x$ - and $y$-axes in the horizontal plane, we are free to choose their orientation to make Equations (5) as simple as possible. Thus we set $\theta=0$; this means that both the $y$-axis and the axis of the lens lie in the vertical plane $x=0$ (see Fig. 2), and that $b$ is the horizontal distance, measured in this plane, from the camera to the plane of motion (see Fig. 7). Moreover, since we install our cameras so that the angle of rotation $\rho$ (see Fig. 2c) is as small as possible, it is a good approximation to set $\rho=0$. With these simplifications, Equations (5) reduce to

$$
\begin{gathered}
X=\frac{\xi}{\cos \phi+\zeta \sin \phi} \\
Z=\frac{-\tan \phi+\zeta}{1+\zeta \tan \phi}
\end{gathered}
$$

which can be used in Equations (10).

The most important result of this theory can be illustrated by a simple example in which the glacier motion, the $x$ - and $y$-axes, and the axis of the lens are all in the horizontal plane (Fig. 7). In this case not only $\theta$ and $\rho$ but also the inclination angle $\phi$ (Fig. 2b) is zero, and it therefore follows from Equations (10a) and (11a) that

$$
u=b\left(1+m^{2}\right)^{\frac{1}{2}} \frac{1}{(1-m \xi)^{2}} \frac{\mathrm{d} \xi}{\mathrm{d} t} .
$$

Equation (12) shows that the glacier speed $u$ is not related to the film-plane speed $\mathrm{d} \xi / \mathrm{d} t$ by a constant proportionality factor unless $m=0$, which corresponds to movement 


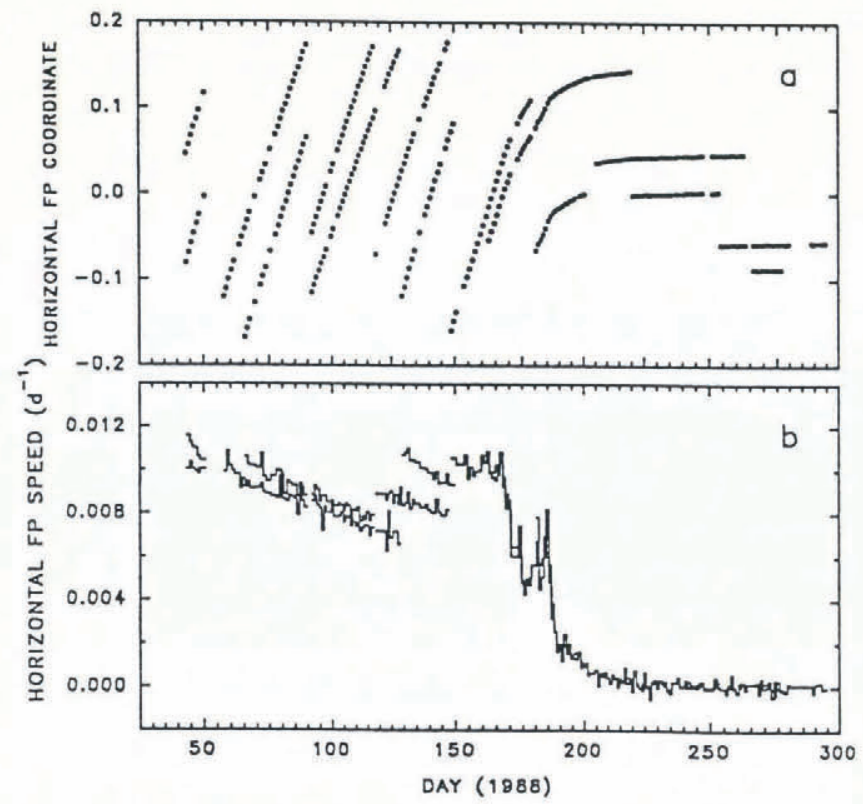

Fig. 8. (a) Horizontal film-plane coordinate $\xi$ for all the moving glacier targets at lower camera. Each curve applies to a different target. (b) Horizontal film-plane speed $\mathrm{d} \xi / \mathrm{d} t$ at lower camera, obtained by differentiation of the curves in (a).

of the target on a trajectory which is perpendicular to the axis of the lens. This means that as a target traverses the field of view its film-plane speed will change even if its actual speed $u$ is constant, unless it moves perpendicular to the axis of the lens. Failure to account for this effect may introduce a substantial systematic error, in the form of an artificial acceleration.

This effect is illustrated with the data from the lower

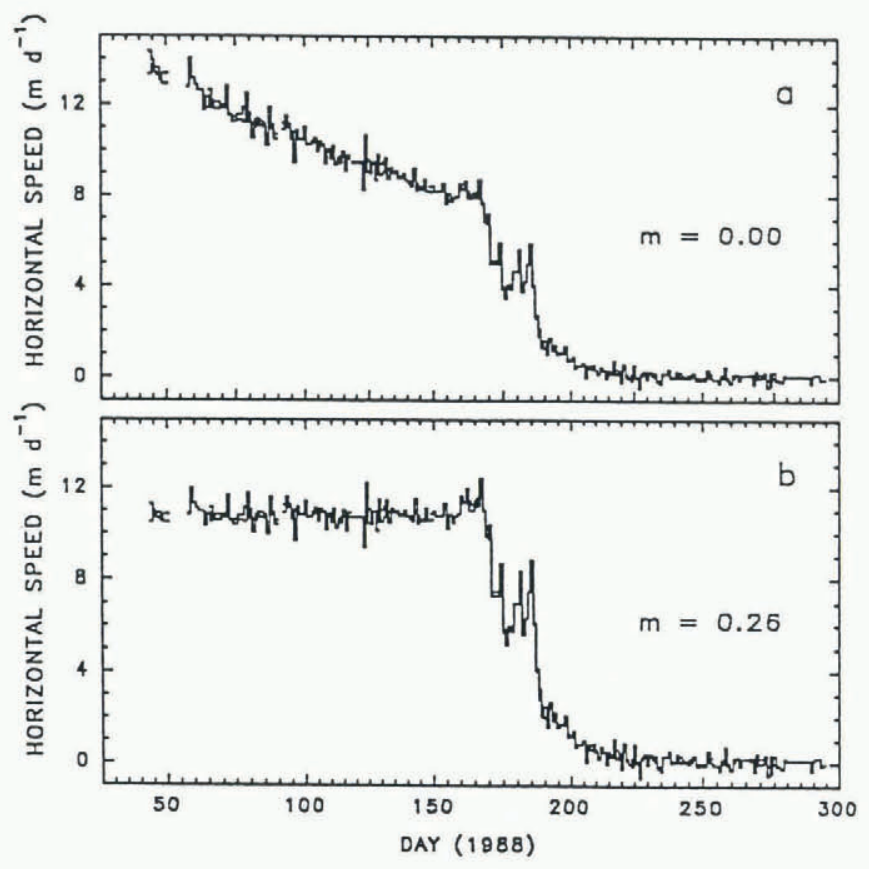

Fig. 9. Horizontal speed $u$ at lower camera, as interpreted for two different values of the quantity $m$ defined by Equation (7) and Figure 7: (a) $m=0$, which corresponds to a $90^{\circ}$ intersection angle in Figure 7. (b) $m=0.26$, which corresponds to a $75.5^{\circ}$ angle (as drawn in Figure 7) and is the best estimate from the field observations. camera on West Fork Glacier (which was rotationally stable), using the measured inclination angle $\phi$ of about $5^{\circ}$ in Equations (11). The horizontal data are shown in Figure $8 \mathrm{a}$, in which each curve expresses the horizontal film-plane coordinate of a particular target versus time. The horizontal film-plane speeds for each target, $d \xi / d t$, obtained by numerical differentiation of the curves in Figure 8a, are shown in Figure 8b. The horizontal speed was assumed to be represented by Equation (10a), with a different value of $b$ for each target, but the same value of $m$ for all. This set of $b$ s was determined, to within an overall scale factor which is the same for all targets, by assuming that the horizontal speed $u$ was identical, in the leastsquares sense, for all pairs of targets overlapping in time. A few data gaps, usually of only a day or two, were filled where necessary to obtain overlap by making linear extrapolations of the horizontal film-plane coordinates based on the five end members of the data set to be extrapolated. The results are shown in Figure 9 for two values of $m$. (The determination of the scale factor, which has been used in Figure 9, is described later.) The first, $m=0$ corresponds to a $90^{\circ}$ angle of intersection between the $x=0$ plane (containing the axis of the lens and the $y$ axis) and the plane of motion of the target. The second, $m=0.26$, corresponds to an intersection angle of about $75.5^{\circ}$, which is our best estimate from the field measurements. These figures show the importance of $m$ in the interpretation of the data.

The quantity $m$ was determined by a compass measurement of the orientation angle $\theta$ of the camera, and of the direction of motion of the glacier near the cameras. The latter measurement was made at lower camera in two ways: first, by measuring the bearing of longitudinal surface features on the surface of the glacier at

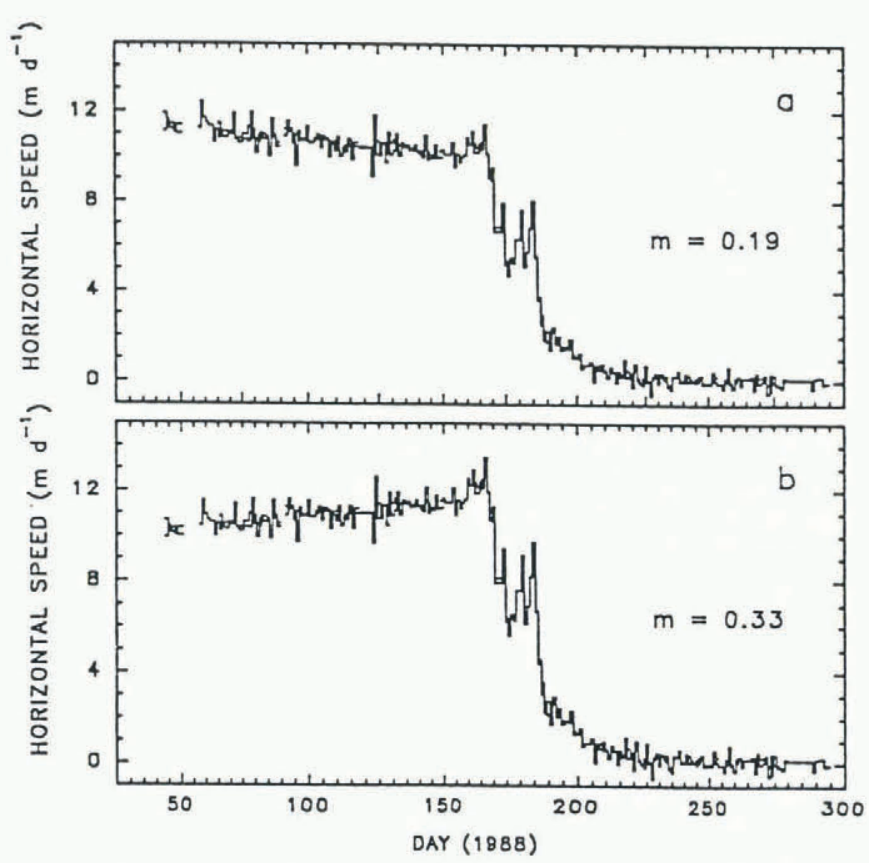

Fig. 10. Horizontal speed $u$ at lower camera, as interpreted for two different values of $m:$ (a) $m=0.19$, which corresponds to a $72^{\circ}$ intersection angle in Figure 7. (b) $m=0.33$, which corresponds to a $79^{\circ}$ angle. These are the two limiting values judged to be consistent with the field observations. 
one time during the surge, and secondly, by mapping the positions of surface features before and after the surge using aerial photographs. The other angles, $\theta$ and $\rho$, were also measured, with the help of a hand level. ( $\rho$, as already noted, was close to zero, and $\phi$, about $5^{\circ}$.) The uncertainty in our estimate of $m$ is about \pm 0.07 at lower camera, which corresponds to an uncertainty of about $\pm 3.5^{\circ}$ in the intersection angle. The horizontal speed in these limits is shown in Figure 10. They show the systematic uncertainty in the speed resulting from the uncertainty in $m$.

The scale factor was determined by surveying, using standard optical methods, a surface feature in the same region as those measured by the camera. The surveying was done on day 112 (21 April) 1988 and was repeated several times over a $1.2 \mathrm{~h}$ time period. This was sufficient to determine the magnitude of the horizontal speed, at least roughly, but not to provide an independent check on its direction. The uncertainty in the scale is about $10 \%$, which is large but acceptable since our primary interest is variations in speed.

A sufficient requirement for the validity of our procedures is that there be no spatial variation in the horizontal speed or its direction (as specified by $m$ ) within the neighborhood of the targets. The errors introduced by transverse and longitudinal velocity gradients have been considered and appear to be small.

\section{THE PROBLEM OF AN ADEQUATE NUMBER OF REFERENCE POINTS}

We have seen that, in general, at least two fixed reference points are required to make corrections for camera instability. In our experience, however, it is often extremely difficult to find, or to install and maintain, even one point, particularly in winter. For this reason, it is worth looking into the requirement of fixed points in more detail.

The discussion is the same as in section III except that we use an approximate version of Equations (5) which gives a clearer physical picture of the role of fixed points. Assume, as we did in section III, that the orientation of the coordinate system is chosen such that $(\theta, \phi, \rho)=(0,0,0)$ at the time of exposure of the first frame. If the instability is not too severe $(\theta, \phi, \rho)$ will be small at the times of exposure of subsequent frames and it will be justified to use small-angle approximations for the trigonometric functions in Equations (5), which, when all second-order terms in the angles are dropped, become

$$
\begin{aligned}
& X \cong \xi+\left(1+\xi^{2}\right) \theta-\xi \zeta \phi+\zeta \rho \\
& Z \cong \zeta+\xi \zeta \theta-\left(1+\zeta^{2}\right) \phi-\xi \rho .
\end{aligned}
$$

Another approximation is valid when $(|\xi|,|\zeta|) \ll(1,1)$, which, by Equations (2), is always true when the camera has a narrow field of view, in the sense that the frame size is much less than the focal length. Then the second-order terms in $(\xi, \zeta)$ can be neglected and Equations (13) become

$$
\begin{aligned}
& X \cong \xi+\theta+\zeta \rho \\
& Z \cong \zeta-\phi-\xi \rho .
\end{aligned}
$$

The final step is to neglect the third terms on the righthand side, which are of second order. Equations (14) then become

$$
\begin{aligned}
& X \cong \xi+\theta \\
& Z \cong \zeta-\phi .
\end{aligned}
$$

The angle $\rho$ does not appear in Equations (15), which means that only two angles $(\theta, \phi)$ are needed to describe the effects of camera instability. Therefore, only one reference point is required to make the necessary corrections. The key question is, when is this true in practice? For the West Fork Glacier upper camera, we have found that Equations (14) are sufficiently accurate to use for the instability corrections, but it is doubtful whether Equations (15) are. This is because, as seen in Figure 3, $\rho$ is much larger than the other angles. Also, because $(|\xi,| \zeta \mid)$ can be as large as $(0.18,0.12$ ) (as determined by the frame size and the focal length by Equations (2)), the second terms on the righthand side of Equations (14) may not be negligible, and Equations (15) may be invalid. However, we have seen other cases in which they do seem to be valid. One can only conclude at this point that the quality of the fixed-point control that is needed depends upon how stably the camera is mounted, how far the glacier moves between frames, the focal length and where the target is located in the field of view.

\section{CONGLUSION}

The purpose of this paper is to show how, with a bare minimum of effort in the field, a reasonably accurate record of speed can be extracted from noisy uncontroled time-lapse camera data. In retrospect, the main difficulty in the West Fork Glacier example was not the lack of essential field data but the effort required to develop the necessary data-reduction procedures, and to apply them to the data. It is not surprising that when a surge, such as that of West Fork Glacier, occurs unexpectedly, the field measurements will by necessity be limited. However, it is our experience that in most situations the interesting glacier observations cannot be carried out under ideal conditions; the unfavorable conditions are the normal ones. Cameras are often unstable and there may be no place to put fixed reference points no matter what resources are available. It therefore seems that our procedures for a surge-type glacier may be useful in other situations.

\section{ACKNOWLEDGEMENTS}

We are very grateful to A. Rasmussen for his careful reading of the manuscript and many valuable suggestions, which came from the perspective of experience with similar glacier problems, and to $H$. Brecher and D. Merchant for their sound advice, which came more from the perspective of professional photogrammetry.

This work was supported by the U.S. National Science Foundation by grants DPP-8519110, DPP8920527, DPP-8822624 and DPP-8822584. 


\section{REFERENCES}

Echelmeyer, K. and W. Harrison. 1989. Surge of West Fork Glacier, Alaska, U.S.A. (Abstract.) Ann. Glaciol., 12, 212.

Flotron, A. 1973. Photogrammetrische Messung von Gletscherbewegungen mit automatischer Kamera. Schweizerische Zeitschrift für Vermessung, Kulturtechnik und Photogrammetrie 1/73.

Harrison, W. D., C. F. Raymond and P. MacKeith. 1986. Short period motion events on Variegated Glacier as observed by automatic photography and seismic methods. Ann. Glaciol., 8, 82-89.
Krimmel, R. M. and L.A. Rasmussen. 1986. Using sequential photography to estimate ice velocity at the terminus of Columbia Glacier, Alaska. Ann. Glaciol., 8, 117-123.

Press, W. H., B. P. Flannery, S. A. Teukolsky and W.T. Vetterling. 1986. Numerical recipes; the art of scientific computing. Cambridge, Cambridge University Press.

Wolfe, P. R. 1983. Elements of photogrammetry. Second edition. New York, McGraw-Hill.

The accuracy of references in the text and in this list is the responsibility of the authors, to whom queries should be addressed. 